\title{
Film als vielschichtiges Medium im Fremdsprachenunterricht *
}

\author{
Gamze Karbi (iD), Ankara - Ayten Genç (iD), Ankara \\ dof $\mathrm{https://dx.doi.org/10.37583/diyalog.} 845598$
}

\begin{abstract}
Deutsch)
In dieser Arbeit soll der Einsatz von Filmen als audiovisuelles Unterrichtsmaterial im Fremdsprachenunterricht erörtert werden. Bei räumlichen und kulturellen Entfernungen zum Zielsprachenland ermöglicht Filmmaterial sprachliche und landeskundliche Inhalte in den Unterricht zu bringen. In diesem Rahmen bieten Filme, die Alltagssprache und Alltagsthemen des Zielsprachenlandes präsentieren, vielfältige Einsatzmöglichkeiten zur Erweiterung der sprachlichen und kulturellen Kompetenzen von Lernenden. Kein anderes Medium verfügt über ein so hohes Potential, die Neugierde der Lerner auf das Zielsprachenland und seine Menschen zu wecken, die Lerner zu motivieren, Emotionen auszulösen und Sprech- und Diskussionsanlässe zu schaffen. Der Einsatz von Filmen leistet einen wichtigen Beitrag zur Gestaltung von effizientem Fremdsprachenunterricht.
\end{abstract}

Schlüsselwörter: Einsatz von Filmen im DaF-Unterricht, audiovisuelle Unterrichtsmedien, Sprachlehrfilme, kulturelles Lernen, sprachliche Fertigkeiten, Fremdsprachendidaktik.

\section{Abstract (English)}

\section{Film as a multifaceted medium in foreign language teaching}

After an overview of the teaching materials, this study examines the use of films, which are an audiovisual media element, in foreign language classes. It is known that as the geographical and cultural distance to the country where the target language is spoken increases, it becomes difficult to transfer some content. Since films contain everyday life and language of the target culture, they are a rich teaching and learning material in foreign language classes. The use of films in foreign language classes has an important contribution to the language classes as films have a high potential to raise curiosity about the country where the target language is spoken and to people who speak that language, in order to increase learning motivation, to reveal feelings, and to prepare the ground for speaking and discussion activities.

Keywords: Foreign Language Teaching, use of films, audio-visual teaching material, cultural learning, language skills.

* Der vorliegende Beitrag stellt einen Ausschnitt aus der Dissertation der Erstautorin dar. 


\section{EXTENDED ABSTRACT}

With transition from the industrial society to the information society, not only business life but also media and communication habits have changed. It is not possible for the field of education to remain unresponsive to the effects of the rapid changes in information and communication technologies on our daily lives. The impact of the media and information age on students of different ages cannot be ignored. Sass (2007: 6) emphasizes that we have been transitioning from a reading-centered world to an audio-visual period, while Ballstaedt (2004: 7) argues that the visual and auditory perception rates of today's youth have increased by $30 \%$ compared to twenty years ago.

When all these changes are taken into account, it is expected that foreign language classes will be designed considering the media usage habits of students in their daily lives. The effective use of films as course material in foreign language classes may contribute to increasing the efficiency of the course. It is known that as the geographical and cultural distance to the country where the target language is spoken increases, it is difficult to transfer some content. The use of films may facilitate the coverage of linguistic and cultural topics in class. Films have a great potential in terms of arousing curiosity, increasing learning motivation, revealing feelings, and preparing the ground for speaking and discussion activities about the target language and the people speaking it (see Wandel, 2002: 139; Schreier, 1999: 77; Hecht, 2003: 49; Lay, 2009: 2). According to Krumm (1998: 523), learning a foreign language means learning a foreign culture; therefore, within the context of intercultural learning, topics aiming at increasing knowledge about the target country and culture are indispensable in foreign language classes. The use of films as teaching materials serves this purpose. Since films include everyday life and daily language, they are a rich teaching and learning material in language classes.

In this study, after an overview of digital and non-digital teaching materials, the use of films, which are an audio-visual media element, as teaching materials is thoroughly examined. Films are the basis of audio-visual media elements and should be considered as a type of text. The elements of the language used in films should be understood in order to understand films. Film analysis does not mean the analysis of the language of the film; it means recognizing the techniques and elements used in films to communicate with the audience; these are contextual, visual, dramatic, formal and aesthetic elements. In this context, the basic model of film analysis developed by Faulstich (2008: 26) is discussed. According to this model, subject, character, building blocks of the film, and meaning/message should be examined respectively. Thaler (2007: 10) listed the advantages of using films in foreign language classes. The advantages of films are that they are motivating, audio-visual, and original; they contribute to the development of all language skills; and they help students acquire and improve their knowledge about the foreign culture. In addition to these advantages, the most important difference that distinguishes films from other teaching materials is that they arouse some feelings in the audience. Decke-Cornill/Küster (2010: 45) demonstrated the efficiency of learning processes in which feelings are included. There are studies showing that emotions play a role in the processing and permanence of knowledge. We first compare what we see, hear and feel with our own experience. By 
stating that what people are interested in, it is not the data or events, but emotions and stories, especially those about people. Spitzer (2002: 160) highlighted that films are not real, yet they interpret the facts. Given the foreign language classes, it can be said that films that tell a story will affect students and lead to the arousal of feelings. This will create writing and speaking environments for students, and they will have the opportunity to talk about the story, people, and their feelings and thoughts. 


\section{Einleitung}

Ein zeitgemäßer Fremdsprachenunterricht darf die Tatsache, dass unser Alltag zunehmend von Medien geprägt wird, nicht einfach ignorieren, denn die Verbreitung der digitalen Medien hat natürlich auch Auswirkungen auf die Lerner ${ }^{1}$ und das Lernen. Nach Joachim von Gottberg (2007: 395) ist „die Einschätzung der Bedeutung der Medien im Erziehungs- und Bildungsprozess abhängig von Grundpositionen und eigenen Medienerfahrungen“. Er unterteilt diese in „Medienpessimismus“, „Medienoptimismus“ und „Medieneuphorik“. Manche Pädagogen warnen vor einer Reizüberflutung der Heranwachsenden und sind der Auffassung, dass sinnvolle Tätigkeiten durch den Medienkonsum verdrängt würden. Ein großer Teil der Probleme, die Jugendliche heute haben und die in der Schule sichtbar werden, zum Beispiel Aggression, Lernunlust, Leseverweigerung, Freizeit und Fun-Mentalität, werden mit den Massenmedien, vor allem mit der Film- und Fernsehkultur, in Verbindung gebracht. Die Wirkung von Film und Fernsehen wird verallgemeinert und manchmal sogar zu einem Feindbild für die Schule gemacht (vgl. Zeitlinger 2003: 78). Optimisten dagegen sehen in den Medien langfristig eine wichtige Voraussetzung für Lern- und Bildungsprozesse (vgl. Wegener 2007: 459). Sie sind sich bewusst, dass die meisten Kinder schon bei Schulantritt und erst recht, wenn sie in höhere Schulstufen kommen, eine reichhaltige mediale Erfahrung haben. Dies macht Lernende in Bezug auf Medienkompetenzen $\mathrm{zu}$ inhaltlichen Experten. Nicht umsonst bezeichnet man die heutige Lernergeneration, die in der digitalen Welt aufgewachsen ist, als „digital natives“. Diese Tatsache sollte nicht übersehen, sondern als Chance für den Unterricht genutzt werden.

Die Verwendung von audiovisuellen Medien im Unterricht wird mit der stetig steigenden Medienpräsenz im Alltag immer wichtiger und erfordert vom Unterrichtenden eine Auseinandersetzung und Beschäftigung mit den Möglichkeiten und Grenzen dieser Medien. Der lateinische Fachbegriff audiovisuell wird aus zwei Bestandteilen zusammengesetzt, die beim Erlernen einer Fremdsprache wichtig sind, Hören und Sehen. Natürlich sind „Hören und Sprechen äußerst eng miteinander verbunden, denn bei der Wahrnehmung gehören Bild und Ton zusammen“ (Ehnert 2001: 1093). Mit audiovisuellen Medien werden Sprache, Geräusche und Musik mit Bildern zusammengeführt.

Heutzutage bieten die digitalen Medien viele Vorteile; man kann CDs, DVDs und Videos erneut hören oder sehen, Bild- und Tonsequenzen stoppen, ohne Bild oder ohne Ton zeigen, bestimmte Teile überspringen und kopieren. Die Lehrkraft kann je nach Lernziel bzw. Aufgabenstellung das Medium ,ausschöpfen“. Tschirner (1997: 55) weist daraufhin, dass die neuen Medien aus der Verschmelzung traditioneller Audio-, Video- und Printmedien mit dem Computer entstehen und ,die Digitalisierung der Daten einerseits die gleichzeitige Präsentation und Manipulation von Ton, Bild, Grafik,

\footnotetext{
1 Zugunsten der besseren Lesbarkeit wird in dieser Arbeit auf die Nennung beider Geschlechter verzichtet; stattdessen wird entweder die Pluralform oder maskuline Form verwendet.
} 
Film und Schrift und andererseits den relativ kostengünstigen und schnellen Zugang ermöglicht".

Spricht man von audiovisuellen Medien, dann kommt dem Film eine besondere Rolle zu; sie nehmen einen festen Platz im DaF-Unterricht ein.

Dem Medium kommt eine wichtige Funktion vor allem im Auslandsunterricht zu, da es auf anschauliche Art unterschiedliche Themen, kommunikative Situationen, Textsorten, sprachliche Register usw. vermitteln und dadurch fremdsprachliche und fremdkulturelle Authentizität ins Klassenzimmer transportieren kann. (Storch 1999: 281)

\section{Film als Textsorte}

Die Wurzel aller audiovisuellen Medien ist der Film und sollte auch als Textsorte aufgefasst werden. Filme erzählen eine Geschichte, die in der Vergangenheit, Gegenwart oder Zukunft spielt. „Seine Sprache ist eine Bildsprache, eine Tonsprache, eine Musiksprache und eine narrativ-dramaturgische Sprache“ (Schwerdtfeger 1989: 15) stellt Schwerdtfeger fest und beschreibt die Textsorte Film mit den folgenden Worten:

Die Textsorte Film hat ein spezifisches System von Zeichen. Durch das Zusammenspiel der Zeichen entsteht die Wirkung der Filmaussage. Die Hauptelemente des Textes Film sind das Bild und der Ton. Der Film als Einheit - sein Bildinhalt, seine Geräusche und seine gesprochene Sprache - ruft bestimmte Wirkungen beim Zuschauer hervor. (Ebd.: 16)

Filme werden in einer bestimmten Absicht gemacht, mit dem Ziel, eine Aussage zu erreichen. Die Filmemacher setzen bestimmte Filmzeichen ein, um eine spezielle Reaktion im Betrachter hervorzurufen. Sie gehen dabei vom Vorwissen des Betrachters über die Bedeutung filmischer Zeichen aus, daher sind Filme also auch als ein Zeichensystem zu verstehen. Borstnar (2008: 16) klassifiziert die filmischen Zeichen nach visuellen Zeichen (Bild und Schrift) und akustischen Zeichen (Sprache, Geräusch und Musik). Bestehend aus filmischen Zeichen, kann der Film, so gesehen, als Textsorte bezeichnet werden und ist dennoch „kein Buch“, wie von Schwerdtfeger (1989: 13) hervorgehoben. Aus diesem Grund erscheint es wichtig, die Unterschiede zwischen Film als Unterrichtsgegenstand und Buch, dem meist verwendeten Unterrichtsmedium aufzuzeigen. Die Einzigartigkeit des Filmmediums ermöglicht Übungs- und Arbeitsformen, die kein anderes Medium gestattet. Dies wird durch die Gegenüberstellung von Lehrbuch und Film verdeutlicht (vgl. Schwerdtfeger 1989: 14).

\begin{tabular}{|l|l|}
\hline Lehrbuch & Film \\
\hline Spricht zunächst einen Sinn an & Spricht mehrere Sinne zugleich an. \\
\hline Geschriebene Texte & Gesprochene Sprache \\
\hline Zeichnungen/Fotos (statisch) & Bewegtes Bild (dynamisch) \\
\hline Beschreibung von Sprechsituationen & Eine Beschreibung entfällt, man sieht und hört \\
\hline
\end{tabular}




\begin{tabular}{|l|l|}
\hline & Personen und wie/mit wem/wo sie sprachlich handeln. \\
\hline Geschriebene Dialoge & $\begin{array}{l}\text { Man hört und sieht die Personen sprechen, } \\
\text { einschließlich Rhythmus, Intonation, Zögerungen, } \\
\text { Wiederholungen usw. }\end{array}$ \\
\hline Geräusche werden „gelesen“ & Geräusche werden gehört. \\
\hline Beschreibung und Dialoge nacheinander & $\begin{array}{l}\text { Gleichzeitigkeit von sozialem Geschehen und seinem } \\
\text { Hintergrund: Das Bild des Sprechers und seines } \\
\text { Kontextes gibt wichtige Anhaltspunkte zum } \\
\text { Globalverstehen der Dialoge. }\end{array}$ \\
\hline [---] & $\begin{array}{l}\text { Viele beiläufige Informationen werden gegeben, so } \\
\text { wird z.B. die Umgebung einer Szene mitgezeigt. }\end{array}$ \\
\hline $\begin{array}{l}\text { Zurückblättern bei Nichtverstehen } \\
\text { möglich }\end{array}$ & $\begin{array}{l}\text { Flüchtigkeit der Filmsituation- Zurückgehen erst durch } \\
\text { Videotechnik möglich. }\end{array}$ \\
\hline
\end{tabular}

Tab. 1: Gegenüberstellung von Lehrbuch und Film

\section{Filme verstehen}

„Filme zu sehen ist leicht, Filme zu verstehen ist schwierig“, schreibt Monaco (2009: 1) und ergänzt: „Da sie Wirklichkeit nachahmen, findet jeder Zugang zu ihrer Oberfläche. Doch sie erzählen in ihrer eigenen Sprache, die zu entschlüsseln ein geschultes Auge verlangt. Je mehr einer über Filme weiß, desto mehr teilen sie mit ihm“. Das heißt, dass wie beim Prozess des Lesens, Text und Zuschauer einander bedingen. Zeitlinger (2003: 80) beschreibt das „Lesen“ von Film- und Fernsehtexten als „einen Prozess dynamischer Wechselwirkung zwischen Text und Leser“".

Abraham (2009: 13, 27) geht näher auf die Sprache des Films ein und spricht von vier Ausdrucksebenen: Diese sind Sprache: Monolog, Dialog, Figurenrede, ggf. Erzähler; Bild: Kamera (-einstellung, -bewegung, -perspektive), Licht, Bewegung, Mimik, Gestik; Ton: Musik, Geräusche; Kulisse: Raumgestaltung, Gegenstände (Filmarchitektur, umbauter Raum, Locations, Requisiten). Diese Ausdrucksebenen weisen auf vier „Künste“, in denen der Film seine Wurzeln hat und die dem Zuschauer das „Verstehen“ von Filmen ermöglichen. Gemeint ist die Fähigkeit, Bilder zu lesen, also ihnen verstehend Sinn und Bedeutung zu geben. Nicht nur bei Filmen, sondern auch bei Fotos und anderen bildlichen Darstellungen kommt es darauf an, die Zeichen $\mathrm{zu}$ verstehen und eine „Botschaft“ zu erkennen, nicht nur abgebildete Objekte auf Papier, Bildschirm oder Leinwand. Diese Fähigkeit wird Visual Literacy genannt. Die Rezeption eines Films ist nach Schröter (2009: 11) „eine sinnliche Angelegenheit“ und „Filme werden selektiv wahrgenommen und die Auswahl und Bedeutungsordnung der Wahrnehmungsinhalte erfolgen nach den Interessen und der Einstellung des Betrachters". Das bedeutet, dass jeder Zuschauer seinen individuellen Film sieht, der sich von dem Film der anderen Zuschauer unterscheidet. „Was ich als Betrachter im Film sehe, ist zwar Fiktion, bedeutet aber in der Situation immer auch eine Vergegenwärtigung eigener Lebenserfahrungen, auf die man seine Eindrücke beziehen kann. Durch den Film werden sie aktualisiert." (Ebd.: 12) Filme bedienen sich 
zunehmend einer Symbolik, die aus anderen Medien, Filmen wie Texten, bekannt ist. Sie spielen mit dem kulturellen Wissen der Zuschauer. Man kann also von einer Interaktion zwischen Film und Zuschauer sprechen, wenn von Filmrezeption die Rede ist. Zeitlinger (2003: 80) unterscheidet zudem noch zwischen Rezeption und Aneignung. Mit Aneignung ist über die Rezeption hinaus auch die Übernahme der rezipierten Texte in den lebensweltlichen Kontext der Zuschauer gemeint. Wesentlich ist dabei die Bedeutung, die ein medialer Text für den Rezipienten hat. Auf diese Weise entsteht also in der Interaktion zwischen Filmtext und Zuschauer etwas gemeinsames Drittes - der rezipierte Text -, der nicht mit dem Originaltext im Film identisch ist. Zu den Grundstrukturen von Texten, in diesem Fall Filmen, gehören die Ästhetik, die Narration, die Rhetorik, die implizite Handlungsanweisung (Appellstruktur) und ermöglichen eine Interaktion mit dem Film (vgl. ebd. 81.).

Diese Elemente erweitern Bechthold/Gericke-Schönhagen (vgl. 1991: 7) mit kinetischen Zeichen wie Körpersprache, Gestik, Mimik etc. paralinguistischen Zeichen wie Stimmklang und Betonung. Dadurch, dass sich diese unterschiedlichen Zeichensysteme im Film überlagern und ineinander verschachteln, wird in einem einzigen Filmbild simultan wahrgenommen, was man im herkömmlichen literarischen Text linear wahrnimmt.

\section{Filmwahrnehmung}

An dieser Stelle soll auf die Wahrnehmung von Filmen, ohne die das Verstehen von Filmen nicht möglich wäre, eingegangen werden. Die Wahrnehmung von Filmen läuft über das Auge und das Ohr ab. Nicht immer werden beide Kanäle zu gleichen Teilen beansprucht; oft dominiert ein Kanal. Brandi/Helmling (1985: 9) weisen daraufhin, dass „es zu positiven Interferenzen kommen kann, wenn die Informationsentnahmen sich über den Seh- und Hörkanal ergänzen, zu negativen Interferenzen hingegen, wenn der Sehkanal den Hörkanal behindert und umgekehrt". An dieser Stelle sollen die beiden Übertragungskanäle näher betrachtet werden: Visuelle Präsentation (Bildfolge und Bildbewegung): Durch sie erkennen wir die Kommunikationssituationen, d.h. den Ort, die Handlung, die Personen, die mit jeweils unterschiedlichen Kameraeinstellungen auf dem Bildschirm erscheinen. Akustische Präsentation (Ton): Sie beinhaltet die gesprochene Sprache, Musik, Geräusche und sogar die Stille (vgl. ebd.: 10).

Wir können bei der normalen Bild-Tonabfolge nicht alles mit derselben Präzision wahrnehmen und verarbeiten, d.h. wir entnehmen Informationen, die uns am stärksten stimulieren. Hieraus ergibt sich für den FSU, dass wir durch Aufgaben zur gezielteren Wahrnehmung bei Lernern beitragen können. Mit dem Filmeinsatz im DaFUnterricht wollen wir nicht nur erreichen, dass der Lerner rezeptiv viel wahrnimmt, sondern wir erzielen vor allem, dass er zu einer Produktion in der Zielsprache angeregt wird, die ihn vom passiven Zuschauer zu einem aktiv Beteiligten macht.

Beim Wahrnehmungsprozess, der durch die Aufnahme von Bild und Ton erfolgt, unterstreichen Brandi/Helmling (vgl. 1985: 14), nimmt der Rezipient, in diesem 
Fall der Lerner, die Situation über die beiden Übertragungskanäle Situation wahr. Er sieht die Personen, den Ort, die Zeit und hört dabei Geräusche, Musik und Sprache. Er nimmt die Situation subjektiv wahr, da bei der Wahrnehmung die Vorerfahrungen und das Vorwissen aktiviert werden und diese individuell sind. Für den Rezipienten sind die Bilder nicht voneinander unabhängig, sondern sie stehen in einem Zusammenhang. Durch die Dekodierung der Bildfolge entsteht beim Rezipienten eine Wirkung. Er interpretiert die Situation. Als letzter Schritt steht die sprachliche Produktion, das Ziel des Fremdsprachenunterrichts. Die sprachliche Produktion steht unter dem Einfluss von unterschiedlichem Vorwissen, Wahrnehmung und sprachlicher Voraussetzung jedes Lerners. Durch verschiedene Arbeitsaufträge wird der Lerner zu mündlicher oder schriftlicher Sprachproduktion angeregt.

\section{Filmanalyse}

Unter dem Begriff Analyse ist die ,systematische Untersuchung eines Gegenstandes oder Sachverhalts hinsichtlich aller einzelnen Komponenten oder Faktoren, die ihn bestimmen“ zu verstehen (Duden 1990: 58). In Bezug auf Filme heißt das, dass alle Elemente, die ein Film beinhaltet, untersucht werden müssen, und zwar systematisch. Kuchenbuch (vgl. 2005: 23) kritisiert die oft anzutreffenden Vorbehalte gegen die Analyse, gegen das Zergliedern. Er vertritt die Auffassung, dass ohne Analyse kein überschaubarer, rationaler Zugang zur Produktion möglich sei, geschweige denn eine wissenschaftliche Auseinandersetzung damit. Auch Hickethier (vgl. 2007: 2) spricht sich für die Filmanalyse aus und weist daraufhin, dass Filme nur konsumiert werden, ohne dass sich die Zuschauer auf die künstlerischen Ausdrucksweisen einlassen und sich damit auskennen. Im Vergleich zur Literatur, die ein Bestandteil von schulischem Unterricht sei, würden die Strategien zur Filmanalyse kaum vermittelt. Betrachtet man die Ziele der Filmanalyse, wird deutlich, dass dieser Bereich eine größere Rolle im Unterricht einnehmen sollte. Filmanalyse dient „der Sensibilisierung der eigenen Wahrnehmung, der Vervollkommnung der ästhetischen Geschmacksbildung, der Steigerung des ästhetischen Genusses, der Gewinnung von Kenntnissen über die audiovisuellen Medien und der genaueren Beschreibung und besseren Beurteilung von medialen Prozessen“" (ebd.: 3).

Der Filmanalyse liegt die Untersuchung der filmischen Mittel zugrunde. Dabei spielen nach Mikos (2008: 13) „inhaltliche, darstellerische, dramaturgische, erzählerische und ästhetisch-gestalterische Mittel ebenso eine Rolle wie die Kontexte, in die filmische Strukturen und Zuschauer eingebunden sind“. Dabei sind die Aspekte inhaltliche Kohärenz, gestalterische Mittel und kommunikativer Prozess wesentlich. Demnach können Filme auf diesen Ebenen untersucht werden: Inhalt und Repräsentation: Plot und Story, Raum und Zeit, Interaktionsverhältnisse, Situative Rahmungen; Narration und Dramaturgie: Spannung, Komik, Bedrohung; Figuren und Akteure: Personen und Rollen, Identifikation, Empathie und Sympathie, Interaktion; Ästhetik und Gestaltung: Kamera, Licht, Schnitt und Montage; Ausstattung, Ton, 
Musik, Visuelle Effekte und Spezialeffekte; Kontexte: Gattungen und Genres, Intertextualität, Diskurse, Lebenswelten, Produktion und Markt (vgl. ebd.: 13).

Von Faulstich (2008: 26) wurde eine Art Grundmodell der Filmanalyse entwickelt, wonach vier verschiedene Zugriffe auf den Film unterschieden werden: Erstens geht es um das WAS im Film, um die Handlung. Was geschieht im Film in welcher Reihenfolge? Zweitens steht das WER im Vordergrund. Welche Figuren oder Charaktere spielen im Film eine Rolle? Drittens wird nach dem WIE gefragt. Welche Bauformen des Erzählens werden im Film verwendet? Und viertens schließlich gipfelt die Analyse im WOZU, in der Frage nach den Normen und Werten, der Ideologie der Message des Films (siehe Abb. 1)

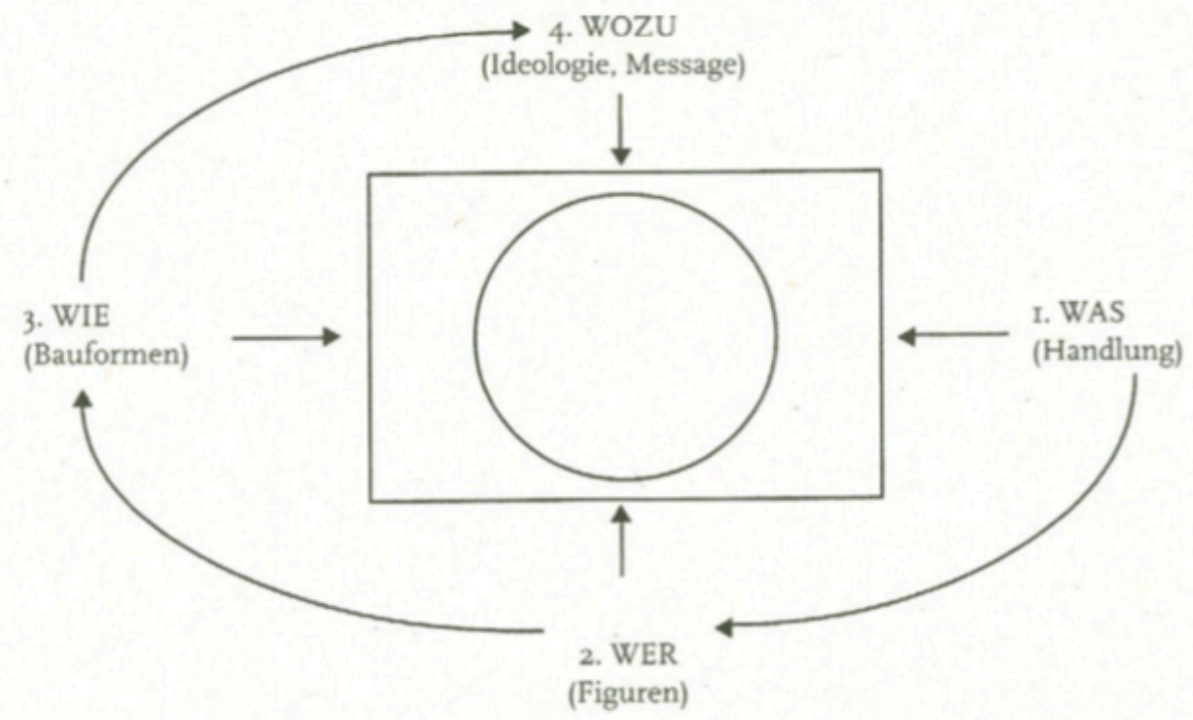

Abb. 1: Grundmodell der Filmanalyse

Nach Biechele (2006: 316) sollten „filmische Darstellungsmittel und deren Wirkungen im FSU thematisiert werden. Beobachtungsaufgaben zur Realisation von Inhalt, Reflektionen zur Umsetzung des Inhalts mit Mitteln der Filmsprache sollten Teil der Arbeit mit Filmen im Fremdsprachenunterricht sein und sind damit Schritte zur Filmanalyse“. Raabe (vgl. 1997: 150) hebt hervor, dass die allgemeine Filmanalyse die Perspektive des FSU nicht kenne und nach der Funktion von Sprache im Film frage. Die fremdsprachenunterrichtliche Filmanalyse habe jedoch nach der Funktion von Film für den Fremdspachenerwerb $\mathrm{zu}$ fragen. Borstnar (2008: 14) bespricht Ansätze, Fragestellungen und Methoden der Filmwissenschaft, von denen hier die Bereiche Ansatz und Fragestellung zitiert werden sollen, da diese deutlich machen, wie im FSU an filmspezifischen Aspekten gearbeitet werden kann (siehe Tab. 2).

\begin{tabular}{|l|l|}
\hline Ansatz & Fragestellung \\
\hline Film als ästhetischer Gegenstand & $\begin{array}{l}\text { Was ist schön / gut im Film? Wie ist er } \\
\text { materialästhetisch beschaffen? }\end{array}$ \\
\hline Film als Kunst & \\
\hline
\end{tabular}




\begin{tabular}{|l|l|}
\hline Film als sprachliches und Sprache & Welche Codes verwendet der Film? \\
\hline Film als Medium der Massenkommunikation & $\begin{array}{l}\text { Welche gesellschaftlich relevanten Themen } \\
\text { behandelt der Film explizit oder implizit? }\end{array}$ \\
\hline $\begin{array}{l}\text { Film als Text } \\
\text { Film als literaturähnliches Zeichensystem }\end{array}$ & $\begin{array}{l}\text { Was und wie erzählt der Film? Wie setzt der } \\
\text { Film literarische Vorlagen um oder wie ist sein } \\
\text { Verhältnis zur Literatur? }\end{array}$ \\
\hline $\begin{array}{l}\text { Film als Aufzeichnungs- und Darstellungsmittel } \\
\text { für alle sicht- und hörbaren Objekte und } \\
\text { Ereignisse }\end{array}$ & $\begin{array}{l}\text { Welche Gegenstände behandelt oder } \\
\text { dokumentiert der Film wie? }\end{array}$ \\
\hline $\begin{array}{l}\text { Film als komplexe Struktur von } \\
\text { interdependenten Zeichensystemen }\end{array}$ & $\begin{array}{l}\text { Welche Zeichensysteme verwendet oder } \\
\text { etabliert der Film auf welche Art? }\end{array}$ \\
\hline $\begin{array}{l}\text { Film als Kommunikationsmittel, welches andere } \\
\text { Kommunikationsmittel auf spezifische Weise } \\
\text { kommuniziert. }\end{array}$ & $\begin{array}{l}\text { Was sind filmspezifische Codesysteme und wie } \\
\text { überlagern sie sich mit den afilmischen? }\end{array}$ \\
\hline $\begin{array}{l}\text { Film als Ware } \\
\text { Film als Wirkfaktor }\end{array}$ & $\begin{array}{l}\text { Welcher Film ist kommerziell erfolgreich und } \\
\text { warum? }\end{array}$ \\
\hline
\end{tabular}

Tab. 2: Ansätze der Filmwissenschaft

Die obigen filmwissenschaftlichen Fragestellungen sind für den FSU von Relevanz, in Abhängigkeit vom Sprachkönnensstand, dem Ziel des Unterrichts und der Art des Films.

\section{Didaktischer Nutzen von Filmen im Fremdsprachenunterricht}

Das Medium Film ermöglicht vielseitige und variable Arbeits- und Übungsformen im FSU. Die Tabelle 3 bietet eine Übersicht über die von Thaler (2007: 10) festgehaltenen „Legitimationsargumente“ für den Einsatz von Filmen im FSU.

\begin{tabular}{|l|l|}
\hline Legitimationsargumente & Zitate \\
\hline Popularität und Motivation & $\begin{array}{l}\text { „Video is today’s medium“. } \\
\text { (Sherman 2003: 2) }\end{array}$ \\
\hline Audiovisuelle Attraktivität & $\begin{array}{l}\text { „If it moves they will watch it“. } \\
\text { (Andy Warhol) }\end{array}$ \\
\hline Authentizität & $\begin{array}{l}\text { „People want access to the world of English- } \\
\text { language media: they want to......use these } \\
\text { language products like normal consumers“. } \\
\text { (Sherman 2003: } 2 \text { ) }\end{array}$ \\
\hline Training rezeptiver Kompetenzen & $\begin{array}{l}\text { „To learn to speak to people they must see and } \\
\text { hear people speaking to each other“. (Sherman } \\
\text { 2003: 14) }\end{array}$ \\
\hline (Hör-Seh-Verstehen) &
\end{tabular}




\begin{tabular}{|l|l|}
\hline $\begin{array}{l}\text { Förderung produktiver Kompetenzen } \\
\text { (Sprechen, Schreiben) }\end{array}$ & $\begin{array}{l}\text { „Gerade medial vorgeführter Stoff schafft das } \\
\text { Bedürfnis zu eigenem Meinen, Fühlen, Urteilen } \\
\text { und Handeln“. (Erdmenger 1989: 130) }\end{array}$ \\
\hline Intertextuell-literarische Kompetenzen & $\begin{array}{l}\text { film on book oder book on film } \\
\text { (Surkamp 2004) }\end{array}$ \\
\hline Interkulturelles Lernen & $\begin{array}{l}\text { Der Film als ,,moving picture book“. } \\
\text { (Sherman 2003: 3) }\end{array}$ \\
\hline $\begin{array}{l}\text { media/film literacy } \\
\text { Formal ästhetische und inhaltlich-kritische }\end{array}$ & $\begin{array}{l}\text {,...bietet es sich an, die formalen, funktionalen } \\
\text { und ästhetischen Kategorien medialer } \\
\text { Textformate im Sinne von film literacy zu } \\
\text { erschließen“. (Thaler 2007) }\end{array}$ \\
\hline
\end{tabular}

Tab. 3: Legitimationsargumente für den Filmeinsatz

Zusätzlich zu den oben aufgeführten Argumenten, die für den Einsatz im FSU sprechen, findet man bei Storch (vgl. 1999: 281) weitere Gründe, die für den Einsatz von Filmen im Unterricht sprechen; demnach können Filme:

- $\quad$ informieren, z.B. landeskundliches Wissen über das Zielsprachenland vermitteln; es können auch ganz aktuelle Ereignisse aufgenommen und in den Unterricht integriert werden;

- authentische Kommunikation in unterschiedlichen kommunikativen Situationen präsentieren und somit authentische Äußerungsanlässe schaffen;

- rezeptive Fertigkeiten fördern, indem Hör-Seh-Verstehen trainiert wird und Bilder als Semantisierungshilfe dienen;

- $\quad$ zum Einüben von sprachlichen Fertigkeiten eingesetzt werden;

- $\quad$ das Interesse der Lernenden wecken und die Lernmotivation fördern.

Die Vorteile des Einsatzes von Filmen im FSU wurden beleuchtet und im Folgenden sollen die Aspekte Emotion, Aufmerksamkeit, Motivation und Authentizität, die durch Filme erzeugt werden und einen Mehrwert für den Unterricht darstellen, näher erörtert werden.

\section{Emotion}

Schwerdtfeger stellt die These auf, dass

Filme und vor allem die mit ihnen verbundenen Übungen und Unterrichtsmethoden es vermögen, ein anderes Gefüge von kognitiven und emotionalen Kräften in den Schülern anzusprechen und eine andere Äußerungsbereitschaft der Schüler zu schaffen, als sie bisher im Fremdsprachenunterricht vorherrschte und fordert die Aufdeckung der Bezüge, die zwischen Emotion und Kognition bestehen, da „die Entdeckung dieser Zusammenhänge in Konsequenzen einmündet für einen Fremdsprachenunterricht, der Filme ernst nimmt und einsetzt. (Schwerdtfeger 1989: 27)

Um einen Überblick über die Faktoren, die beim Lernen eine Rolle spielen zu gewinnen, wird folgende Abbildung herangezogen, denn Lernen ist abhängig von 
kognitiven, motivationalen, affektiven und sozialen Bedingungen (vgl. Kieweg 2003: $6)$.

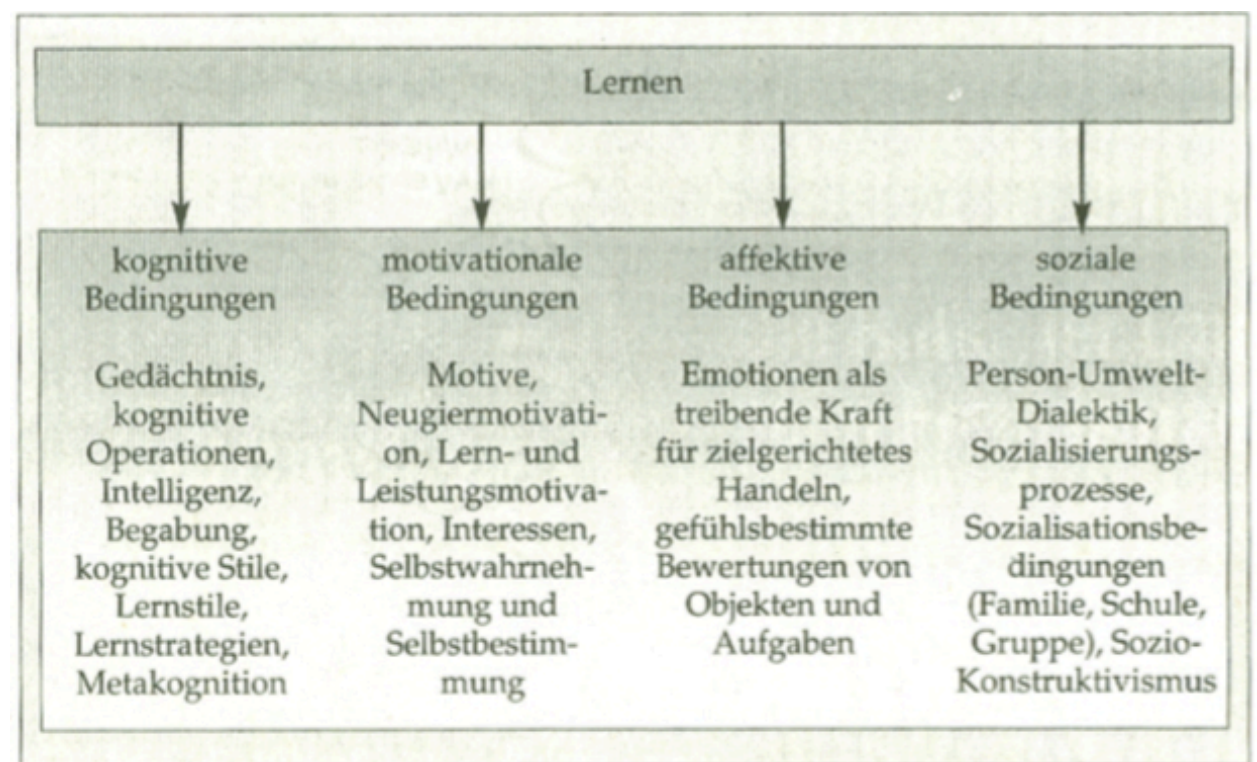

Abb. 2: Lernfaktoren

Ansätze des Kognitivismus verstehen Lernen als einen Prozess der Informationsverarbeitung. Affektive Faktoren wie Emotionen spielen dabei nur eine untergeordnete Rolle. Neuere neurophysiologische Studien jedoch machen deutlich, dass Emotionen einen immensen, sehr wohl auch positiven Einfluss auf das Lernen haben (vgl. Decke-Cornill/Küster 2010: 45). Dieter Wolff (2004: 94) vermutet, dass „wir nur das aus unserer Umwelt verarbeiten, worauf uns unsere Emotionen verweisen“. Nicht nur die Initiierung von Lernprozessen, sondern auch und gerade ihr Verlauf sei in hohem Maß abhängig vom Einfluss der Emotionalität. Kieweg (2003: 7) weist daraufhin, dass ,positive Gefühlsbetonungen nicht nur die Menge der Informationsverarbeitung, sondern auch die Dauerhaftigkeit von Gedächtnisleistungen erhöhen“. Die Aussage Wolffs (vgl. ebd.: 98), dass eine tiefer gehende Verarbeitung im Sinne eines Behaltens einer Aussage nur stattfinde, wenn der Sprachverarbeiter emotional involviert sei, wenn er den Verarbeitungsprozess als bedeutsam für seine eigene Person erachte und wenn er sich mit der Aussage identifiziere, spricht für den Einsatz von Filmen im FSU. Es ist erwiesen, dass wir alle Reize, die wir hören, sehen oder fühlen, als erstes mit unseren Vorerfahrungen verknüpfen. Spitzer (2002: 160) sagt dazu: „Was den Menschen umtreibt, sind nicht Fakten und Daten, sondern Gefühle, Geschichten und vor allem andere Menschen. Filme sind zwar nicht Wirklichkeit, aber sie interpretieren die Wirklichkeit". Bedeutend für den Fremdsprachenunterricht ist es, dass Filme Geschichten über den Menschen erzählen und diese Geschichten können aus dem Erfahrungsbereich der Lerner sein oder sie auf irgendeine Weise berühren, was einen guten Ausgangspunkt für authentische Sprechanlässe darstellt: Filme wecken Emotionen der Lernenden und sie äußern sich über die Geschichte und ihre Gedanken und Gefühle dazu. 


\section{Aufmerksamkeit}

Gutenberg-Galaxy ist ein von Marshall McLuhan (1962) übernommener Begriff und bezeichnet eine vom Medium Buch geprägte Welt. Diese Welt ist im Wandel. Im Alltag „sehen“ wir mehr, als dass wir lesen. Dieses Phänomen hat eine Folge: Kinder bilden ihre Vorstellungen davon, was Erzählen und Erzähltes ist, weniger am Buch bzw. Printmedien als am Film bzw. an AV-Medien aus (Frederking\&Krommer\&Maiwald 2008: 143). Dieser Auffassung ist auch Sass (2007: 6); sie hebt hervor, dass wir ,in einer Zeit des Umbruchs von einer sprach- zu einer bildzentrierten Kultur leben und Jugendliche heute eine 30 Prozent höhere visuelle und akustische Wahrnehmungsgeschwindigkeit als vor zwanzig Jahren haben“. Die Rezeptionsbeschleunigung bedeutet nicht, dass automatisch ein Lernprozess stattfindet, denn ohne die gezielte Auseinandersetzung mit den Reizen passiert nichts. Es ist erwiesen, dass unser Gehirn nur Interessantes und Neues aufnimmt und es speichert (vgl. Spitzer 2002: 155). Im Unterricht können wir die Aufmerksamkeit der Lernenden durch eine interessante Unterrichtsgestaltung gewinnen. Wenn wir etwas sehen und gleichzeitig hören, dann ist die Chance der Wahrnehmung und der Verarbeitung in Wissen relativ hoch. Mit dem Aufmerken beginnt das Merken (vgl. Ballstaedt 2004: 4). Die Forschung unterscheidet zwei Arten von Aufmerksamkeit: die unwillkürliche und die willkürliche. Ballstaedt (2004: 4) definiert die unwillkürliche Aufmerksamkeit als „die automatische Zuwendung zu unerwarteten Reizen, also das, was wir im Alltag ständig erleben: Wir sehen eine grelle Leuchtreklame und schauen unwillkürlich dorthin, auch wenn uns die Inhalte nur wenig interessieren“. Die willkürliche Aufmerksamkeit hingegen ist die ,intentionale Zuwendung $\mathrm{zu}$ bestimmten Inhalten; jeder pickt sich aus dem Angebot heraus, was ihn speziell interessiert" (ebd.).

Hierbei sollte beachtet werden, dass die willkürliche Aufmerksamkeit zeitlich begrenzt ist, deshalb ist wichtig, im Unterricht interessante und neue Inhalte zu zeigen; hier ist Film ein sehr brauchbares Medium. Übertragen auf den Fremdsprachenunterricht heißt das, dass wir die willkürliche Aufmerksamkeit steigern können, indem wir die Lernenden ansprechendes audiovisuelles Material für den Fremdsprachenunterricht auswählen und die Lernenden sich durch Beobachtungsaufgaben und kreativen Arbeitsformen mit dem Filmmaterial beschäftigen. Somit wird die Aufmerksamkeit geweckt, sie befassen sich mit dem Unterrichtsinhalt und aktivieren ihr Vorwissen. Kurz gesagt: sie lernen.

\section{Motivation}

In Bezug auf das Erlernen von Fremdsprachen definiert Riemer (2010a: 219) den Begriff Motivation, als ,ein affektives Lernermerkmal, dem ein wesentlicher Einfluss auf den Erfolg und die Schnelligkeit des Lernens einer Zielsprache zugeschrieben wird“. In der Motivationsforschung hat sich generell die Unterscheidung von intrinsischer und extrinsischer Motivation als grundlegend erwiesen (vgl. DeckeCornill/Küster 2010: 47). Bei der extrinsischen Motivation brauchen Lernende Anreize, die von außen kommen, die intrinsisch Motivierten hingegen haben Interesse und Spaß, 
eine Zielsprache zu lernen; es ist eher ein inneres Bedürfnis. Huneke/Steinig (2010: 20) weisen darauf hin, dass Motivation als Einflussfaktor schwer zu operationalisieren sei, da es schwierig sei festzustellen, „ob ein Lerner deshalb so erfolgreich eine Fremdsprache lerne, weil er hoch motiviert sei oder ob er hoch motiviert sei, weil er so gute Fortschritte mache“. Auch Riemer (2010a: 219) stellt fest, dass Motivation nicht direkt beobachtbar sei, zwischen Individuen variiere und Schwankungen unterliege. Obwohl Motivation multidimensional und dynamisch ist, können Konsequenzen für die Praxis des FSU im Sinne von Motivierung hervorgehen. Jeder Lerner hat eine andere Motivation, eine Fremdsprache zu erlernen. Lehrkräfte können jedoch durch Motivierung im Unterricht zum Lernerfolg beitragen. Demnach impliziert Motivierung nach Riemer (2010b: 172) „,ie gezielte Auswahl an den Interessen und Bedürfnissen ausgerichteter Unterrichtsgegenstände, Materialien, Medien und Lehrtechniken“. Diese Auffassung wird auch von Neuner et al. (vgl. 2009: 121) unterstützt, indem festgehalten wird, dass motivierende Materialien wie z.B. Spiele, Bilder, Filme, Musik und authentische Texte das Sprachenlernen interessant machen und emotionale Zugänge öffnen können. Übertragen auf den Kontext dieser Arbeit, ist die Schlussfolgerung, dass es sich bei Filmen um authentisches interessantes Material handelt, das eine motivierende Funktion auf die Lernenden hat.

\section{Authentizität}

Der kommunikative FSU forderte schon in den 1980er Jahren authentische Kommunikationsanlässe im Unterricht. Auch im konstruktivistischen Ansatz ist die Authentizität von Unterrichtsinhalten ein wichtiges Prinzip (vgl. Mohr 2010: 21). „In methodisch-didaktischen Texten werden diejenigen Materialien als authentisch bezeichnet, zu denen Lerner in ihrem Alltag vorrangig Zugang haben; Rezeptionsgewohnheiten und Unterhaltungsbedürfnisse der Lerner sind so ebenfalls ein Kriterium für Authentizität und für die Auswahl von Materialien für das unterrichtliche Sprachenlernen“ (vgl. ebd.: 21). Wichtigstes Unterrichtsmaterial im FSU ist und bleibt das Lehrwerk. Insbesondere außerhalb des Zielsprachenlandes stellen die in Lehrwerken präsentierten Themen und Inhalte den Hauptzugang zur fremden Sprache und Kultur dar. Was die Forderung der Landeskunde nach Aktualität und Authentizität betrifft, kann ein Lehrwerk jedoch nicht mit elektronischen Medien konkurrieren (vgl. Krumm 2001: 1030). Aus diesem Grund wird unter den Punkten für einen Einsatz von Filmen im FSU immer wieder die Authentizität ins Feld geführt (vgl. Thaler 2010: 142). Im Folgenden lohnt es sich auch einen Blick auf die Leistungsfähigkeit von Sprachlehrfilmen zu werfen.

\section{Authentischer Film versus Sprachlehrfilm}

Neben Spielfilmen gibt es auch Sprachlehrfilme, die für ein bestimmtes Publikum geplant und produziert sind. Dieses Filmmaterial ist nicht authentisch, denn Sprache wird progressionsbezogen gebraucht um neue Vokabeln, Strukturen oder Redemittel 
einzuführen. Verfolgt wird ein festes Lernziel, das sich auf Sprachstrukturen oder kommunikative Fähigkeiten bezieht. Lonergan (1987: 18) weist daraufhin, dass Sprache und Situationen in diesen Filmen meistens gut zu Lehrbüchern und Lehrplänen passen und sowohl mit, als auch ohne gedruckte Begleitmaterialien eingesetzt werden können. Da Sprachlehrfilme verschiedene didaktische Möglichkeiten bieten, sind sie für eine Lehr-/Lernsituation attraktiv. Erdmenger (1997: 133) unterscheidet dabei zwischen Sprachlehrfilmen mit sprachlichem und landeskundlichem Schwerpunkt.

An dieser Stelle soll ein Projekt vorgestellt werden, das den Einfluss von authentischem Filmmaterial auf Interesse und Lernerfolg untersucht hat. Das Saarbrückener Forschungsprojekt (vgl. Bufe/Deichsel/Dethloff, 1984) zum Hörverstehen im fernsehunterstützten FSU (Französisch) liefert diesbezüglich wichtige Erkenntnisse. Das Saarbrückener Projekt untersuchte mittels Kurz- und Langzeitstudien die unterschiedlichen Wirkungen eines fernsehgestützten und eines lediglich mit auditivem Zusatzmaterial arbeitenden FSU. Die Testgruppen bestanden aus Französisch-Studenten der Universität Saarbrücken. Das Testmaterial wurde aus Segmenten authentischer französischer Nachrichtensendungen, Magazine oder Werbespots zusammengestellt. Untersuchungen $\mathrm{zu}$ den medialbedingten Langzeiteffekten ergaben, dass Sprachkenntnisse schneller und dauerhafter gespeichert werden, wenn der Unterricht durch das Fernsehen unterstützt wird (vgl., ebd.: 17). Empirische Studien im Rahmen dieses Projekts belegen, dass die Präferenz der Lernenden für das Medium Film zumindest dann sehr signifikant ausgeprägt ist, wenn systematisch authentische Filme bzw. Sequenzen gezeigt werden. Dieses eindeutige Resultat des Saarbrückener Forschungsprojekts bezieht sich jedoch nicht auf die Motivationswirkung beim Einsatz von didaktischen Filmen. Gemäß den vorliegenden Resultaten ist der motivierende Effekt von sprachdidaktischen Filmen im Vergleich zu Spielfilmen und Kurzfilmen als gering einzustufen (vgl., ebd.: 13).

\section{Forschungsmethode}

In dieser Arbeit wurde als Datenerhebungstechnik die Dokumentenanalyse verwendet, um Videomaterial für den DaF-Unterricht zu sichten und auszuwerten. Das Ziel der Dokumentenanalyse ist es, eine Orientierung im Themenfeld Videomaterial im DaFUnterricht zu ermöglichen, indem vorhandene Materialien recherchiert und analysiert wurden.

Die Untersuchung hat ergeben, dass die Anfänge des Filmeinsatzes im DaFBereich in die 1960er Jahre zurückreichen. Der erste Sprachlehrfilm erschien im Jahr 1966 und trägt den Titel Guten Tag $^{3}$. Das Video-Material, das sich an Anfänger richtet, wurde vom Goethe-Institut entwickelt und im Jahr 1967 folgte ein Begleitbuch mit dem Titel Werkhefte für technische Unterrichtsmittel. Dieses Begleitmaterial enthielt methodisch-didaktische Hinweise für Lehrkräfte, die das Material Guten Tag in ihrem Unterricht einsetzten.

\footnotetext{
${ }^{3}$ Guten Tag, 1966, München: Goethe Institut/Bayerischer Rundfunk.
} 
Mit der kommunikativen Wende in der Fremdsprachendidaktik wurde der Bedarf an authentischen Unterrichtsmaterialien größer. Der Einsatz von Videos im DaFUnterricht ermöglichte es, sprachlich handelnde Menschen in Alltagssituationen in deutschsprachiger Umgebung zu zeigen. Durch die Verbreitung von Videorekordern und der Videokassette schaffte es der Film, sich einen besonderen Platz im FSU zu erobern.

Im Folgenden soll eine Übersicht von Videomaterial, das überwiegend in den 90er Jahren für den DaF-Unterricht entwickelt wurde, gegeben werden.

Hauptstraße $117^{4}$ ist ein Videomaterial für erwachsene Anfänger, das im Rahmen eines Projekts entstanden ist. Die Filme drehen sich um das Leben von Nachbarn in einem Mietshaus in einer deutschen Stadt. Thematisiert werden Probleme, Chancen und Hoffnungen der Mietshausbewohner.

Hallo aus Berlin ${ }^{5}$ ist ein für jugendliche DaF-Lerner der Niveaustufe A1/A2 entwickeltes Videomaterial aus den 90er Jahren. Im Mittelpunkt sind sechs Jugendliche aus Berlin. Die Themen der einzelnen Episoden, die jeweils 15 Minuten dauern, sind Familie, Wohnen, in der Stadt, Essen und Trinken, Schule, Freizeit, Gesundheit, Ferien und Feste, Berlin. Die Folgen werden mit Interviews und Musik präsentiert- unterstützt von Trickfilmsequenzen. Sprachlich orientiert sich das Material am Stoffplan des A1Niveaus. Das Material wird ergänzt durch Spiele, Rätsel und kreative Aufgaben für die Lernenden. Eine Handreichung für Lehrkräfte bietet methodisch-didaktische Hinweise und Kopiervorlagen.

Bei redaktion- $D^{6}$, einem weiteren Video-Sprachkurs, handelt es sich um eine Spielfilmreihe, die speziell für den FSU entwickelt wurde und Zugang zur deutschen Alltagskultur gewährt. Bei den Figuren des Films handelt es sich um Paul und Laura, die zwei Internet-Journalisten sind und mysteriöse Fälle auflösen. Das Material richtet sich an Anfänger und umfasst 12 Folgen zu jeweils 15 Minuten und verfügt über ein Begleitmaterial.

Ein Beispiel für Videomaterial mit landeskundlichem Schwerpunkt stellt die Filmreihe $\mathrm{KuBus}^{7}$ dar. Themen, Ereignisse und Personen aus Deutschland werden in 49 Videos von jeweils 15 Minuten Länge dargestellt. Die Beiträge werden durch einen Hintergrundartikel begleitet, zu den Videos, die sich für fortgeschrittene Deutschlerner eignen, gibt es auch eine Textfassung.

Einblicke $^{8}$ ist ein Videosprachkurs, der auch für Selbstlerner geeignet ist. Das Material setzt sich aus Videokassetten, Audiokassetten, Begleitheften für Selbstlerner und einem Dialogbuch zusammen. In 19 Folgen wird die deutsche Gesellschaft vorgestellt, indem kulturelle, wirtschaftliche, ökologische und politische Themen behandelt werden.

\footnotetext{
${ }^{4}$ Hauptstraße 117 Eine Videoserie aus dem Ruhrgebiet, 1992, München: Goethe Institut.

${ }^{5}$ Hallo aus Berlin, 1996, London: BBC-Education/Goethe Institut.

${ }^{6}$ redaktion-D, 2002, München: Deutsche Welle/Goethe Institut.

${ }^{7}$ KuBus, 1997, Bonn: Inter Nationes.

${ }^{8}$ Einblicke. Ein deutscher Sprachkurs, 1998, München/Bonn: Goethe Institut/Inter Nationes.
} 
Die Generation „Videokassette“ wird heutzutage von Videomaterialien abgelöst, die in Form von DVDs erhältlich sind und von Video-Clips, die online abrufbar sind. Zahlreiche digitale Angebote in unterschiedlichen Formaten sind im Internet zu finden. In den letzten Jahren hat sich Filmmaterial im Unterricht immer stärker durchgesetzt, ein Beweis dafür ist, dass die Lehrwerke mit umfangreichen Begleitmaterialien erscheinen - darunter auch Filmmaterial. Um einige Beispiele zu lehrwerkbegleitendem Filmmaterial zu geben, können die Lehrwerke Menschen, geni@l, studio d, Aussichten, Das Leben, Beste Freunde, die bei unterschiedlichen Verlagen erscheinen, genannt werden. Es hat sich herausgestellt, dass Filmmaterialien, die für ein Lehrwerk entwickelt wurden eine sinnvolle Ergänzung darstellen und bei der Vermarktung von Lehrwerken auch eine Rolle spielen.

$\mathrm{Zu}$ den Themen auf den Niveaustufen A1-B1 gehören z.B. Hobbys, Schule, Wohnen, Einkaufen, Sport, Tagesablauf, Wegbeschreibung, Gesundheit. Die Filmsequenzen erfordern keine fortgeschrittenen Deutschkenntnisse, sondern können schon im Anfängerunterricht eingesetzt werden. Es wirkt motivierend auf Anfänger, wenn sie sehen, dass sie Filmmaterial in der Zielsprache folgen können. Da die oben erwähnten Themenbereiche in allen DaF-Lehrwerken der Anfängerstufe behandelt werden, können die Filmmaterialien auch lehrwerkunabhängig und in beliebiger Reihenfolge eingesetzt werden. Zu den DVDs gibt es meistens Begleitmaterialien, die fertige Arbeitsblätter, Kopiervorlagen und Unterrichtsideen beinhalten. Die Lehrkraft kann das didaktisierte Filmmaterial mühelos einsetzen und muss keine umfangreiche Unterrichtsvorbereitung machen. Auch die Länge von etwa 2-4 Minuten pro Filmsequenz ist praktisch, denn dadurch genügt für den Einsatz schon eine Unterrichtseinheit.

\section{Fazit}

Wir leben in einer digitalisierten Welt, in der sich die Intensität unserer Mediennutzung und unsere Medienpräferenzen stark geändert haben. Medienkompetenzen sind sowohl bei Lehrkräften, als auch bei Lernenden zu einer Schlüssenqualifikation geworden. Der Mensch hat sich in den letzten Jahrzehnten vom „Vielleser“ zum „Vielseher“ entwickelt; wir sind täglich zahllosen akustischen und bildlichen Reizen ausgesetzt, infolgedessen wurde unsere Wahrnehmungsgeschwindigkeit beschleunigt. Hinzu kommt die Tatsache, dass wir es zunehmend mit einer Lernergeneration zu tun haben, die als „digital natives“ bezeichnet wird, also Lernende, die in der digitalen Welt aufgewachsen sind. Ein zeitgemäßer FSU, sollte die Besonderheiten und Bedürfnisse der Lernenden beachten und Schlüsse für die Gestaltung des Fremdsprachenunterrichts ziehen.

Heutzutage sind Medien, insbesondere audiovisuelle Medien ein fester Bestandteil unseres Lebens. Filme werden in Form von Serien und Spielfilmen, egal ob zu Hause oder im Kino konsumiert und gehören zum Alltag. Ein derart beliebtes Medium kann auch den FSU auf vielfältige Weise bereichern. Filme haben ein sehr großes Potential, die Vermittlung von sprachlichen und kulturellen Inhalten im 
Unterricht zu ermöglichen und diese zu erweitern. Keinem anderen Medium gelingt es, in so hohem Maß Zugang zu gesellschaftlichen und landeskundlichen Themen zu verschaffen. Alltagsthemen, Alltagssprache, authentische Kommunikationsanlässe, kulturelle Besonderheiten, Körpersprache, Aussprache und vieles mehr, wird innerhalb weniger Sekunden in den Klassenraum transportiert. Filme wecken Neugierde und Interesse und motivieren die Lernenden. Durch gezielte Übungen und Aufgaben können sprachliche und landeskundliche Fertigkeiten ausgebaut werden.

Die Untersuchung von Filmmaterial, das für den DaF-Unterricht entwickelt wurde hat gezeigt, dass Filme schon sehr früh in den DaF-Unterricht integriert wurden, nämlich seit Mitte der 1960er Jahre. Auch wenn sich die technische Form des Mediums in den Jahren von der Videokassette zur DVD und digital erhältlichen Film-Clips entwickelt und verbreitet hat, ist und bleibt der Film ein beliebtes und unverzichtbares Medium für den FSU. Filme können im Lehrwerk dargebotene Inhalte sinnvoll ergänzen und dazu beitragen, dass Strukturen und landeskundliche Elemente dargeboten und gefestigt werden. Neben dem Einsatz von Sprachlehrfilmen, die für Lehrwerke entwickelt wurden, zeichnet sich auch die Tendenz ab, authentische Filme, also Filme, die nicht für den Unterricht entwickelt wurden, z.B. Spielfilme, Kurzfilme, Werbefilme etc. im Unterricht einzusetzen. Das Medium Film ist angesichts der breiten Auswahl, den Einsatzmöglichkeiten und dem didaktischen Mehrwert für den Fremdsprachenunterricht vielfältig, aktuell und voller Potentiale.

\section{Literaturverzeichnis}

Abraham, Ulf (2009): Filme im Deutschunterricht. Seelze-Velber: Kallmeyer/Klett.

Ballstaedt, Steffen-Peter (2004): Kognition und Wahrnehmung in der Informations- und Wissensgesellschaft, Konsequenzen gesellschaftlicher Veränderungen für die Psyche. In: Kübler, Hans-Dieter et al. (Hg.): Wissensgesellschaft. Neue Medien und ihre Konsequenzen. Bonn: Bundeszentrale für politische Bildung, 621-641.

Biechele, Barbara (1989): Video im Sprachunterricht- zu ausgewählten Problemen der Theorie und Praxis eines neuen Mediums für den Fremdsprachenunterricht. In: Schmigalla, Hans (Hg.): Neuere Entwicklungen im Fach Deutsch als Fremdsprache. Wissenschaftliche Beiträge der FriedrichSchiller-Universität: Jena, 82-97.

Biechele, Barbara (2006): Film/Video/DVD in Deutsch als Fremdsprache - Bestandsaufnahme und Perspektiven. In: Barkowski, Hans/Wolff, Armin (Hg.): Materialien Deutsch als Fremdsprache. Heft 76. Regensburg: Fachverband Deutsch als Fremdsprache.

Borstnar, Nils et al. (2008): Einführung in die Film- und Fernsehwissenschaft. Konstanz: UVK Verlagsgesellschaft.

Brandi, Marie-Luise/ Helmling, Brigitte (1985): Arbeit mit Video am Beispiel von Spielfilmen. München: Goethe-Institut.

Brandi, Marie-Luise (1996): Video im Deutschunterricht. Berlin/München: Goethe-Institut.

Decke-Cornill, Helene/ Küster, Lutz (2010): Fremdsprachendidaktik. Tübingen: Narr 
Ehnert, Rolf (2001): Audiovisuelle Medien. In: Helbig, Gerhard et al. (Hg.): Deutsch als Fremdsprache. Ein internationales Handbuch. Verlag de Gruyter, Berlin und New York, Band 2, 1093-1097.

Erdmenger, Manfred (1997): Medien im Fremdsprachenunterricht. Hardware, Software und Methodik. Braunschweig: Schmidt.

Faulstich, Werner (2008): Grundkurs Filmanalyse. Paderborn: Fink.

Frederking, Volker/ Krommer, Axel/ Maiwald, Klaus (2008): Mediendidaktik Deutsch. Eine Einführung. Berlin: Erich Schmidt.

Hecht, Susanne (2003): Hauptstraße 117. Analyse, Entwicklung und Einsatz unterrichtsbegleitenden Videomaterials im Fremdsprachenunterricht für erwachsene Anfänger-auch als Grundbaustein in Multimedia-Anwendungen. 3. Auflage. Eitorf: Gata.

Hickethier, Knut (2007): Film- und Fernsehanalyse. Stuttgart: Metzler und Carl Ernst Poeschel.

Huneke, Hans-Werner/Steinig, Wolfgang (2010): Deutsch als Fremdsprache. Eine Einführung. Berlin: Erich Schmidt.

Kieweg, Werner (2003): Die Rolle der Emotionen beim Fremdsprachenlernen. In: Der fremdsprachliche Unterricht Englisch, 37 (63), 4-11.

Kuchenbuch, Thomas (2005): Filmanalyse. Theorien. Methoden. Kritik. Wien/Köln/Weimar: Böhlau.

Krumm, Hans-Jürgen (2001): Lehrwerkproduktion, Lehrwerkanalyse, Lehrwerkkritik. In: Helbig, Gerhard et al. (Hg.): Deutsch als Fremdsprache: Ein internationales Handbuch. Berlin: Walter de Gruyter, 1029-1041.

Lonergan, Jack (1987): Fremdsprachenunterricht mit Video. Ein Handbuch mit Materialien. München: Max Hueber.

Mikos, Lothar (2008): Film- und Fernsehanalyse. Konstanz: UVK Verlagsgesellschaft.

Mohr, Imke (2010): Authentizität. In: Barkowski, Hans/Krumm, Hans-Jürgen (Hg.): Fachlexikon Deutsch als Fremd- und Zweitsprache. Tübingen: Narr, 20.

Monaco, James (2009): Film verstehen. Kunst, Technik, Sprache, Geschichte und Theorie des Films und der Medien. Mit einer Einführung in Multimedia. Hamburg: Rowohlt.

Neuner, Gerhard et al. (2009): Deutsch als zweite Fremdsprache. Fernstudieneinheit 26. München: Goethe-Institut.

Raabe, Horst (1997): „Das Auge hört mit“- Sehstrategien im Fremdsprachenunterricht? In: Rampillon, Ute/Zimmermann, Günther (Hg.): Strategien und Techniken beim Erwerb fremder Sprachen. Ismaning: Hueber, 150-172.

Raabe, Horst (2007): Audiovisuelle Medien. In: Bausch, Karl-Richard/Christ, Herbert/Krumm, Jürgen (Hg.): Handbuch Fremdsprachenunterricht. 7. Auflage. Tübingen: Francke, 423-426.

Riemer, Claudia (2010a): Motivation. In: Barkowski, Hans/Krumm, Hans-Jürgen (Hg.): Fachlexikon Deutsch als Fremd- und Zweitsprache. Tübingen: Narr, 219-220.

Riemer, Claudia (2010b): Motivation. In: Hallet, Wolfgang/Königs, Frank G. (Hg.): Handbuch Fremdsprachendidaktik. Seelze-Velber: Kallmeyer/Klett, 168-173.

Sass, Anne (2007): Filme im Unterricht- Sehen(d) lernen. Fremdsprache Deutsch. In: Zeitschrift für die Praxis des Deutschunterrichts, Heft 36, 5-13.

Schreier, Horst (1999): Die Arbeit mit audiovisuellen Medien. In: Raasch, Albert/Bühler, Peter (Hg.): Angewandte Linguistik und Sprachlehrforschung: entdecken, erfahren, erleben. Saarbrücken: Ottweiler, 77-83. 
Schröter, Erhart (2009): Filme im Unterricht. Auswählen, analysieren, diskutieren. Weinheim und Basel: Beltz.

Schwerdtfeger, Inge C. (1989): Sehen und Verstehen. Arbeit mit Filmen im Unterricht Deutsch als Fremdsprache. Berlin und München: Langenscheidt.

Schwerdtfeger, Inge C. (2003): Übungen zum Hör-Seh-Verstehen. In: Bausch, Karl-Richard/Christ, Herbert/Krumm, Jürgen (Hg.): Handbuch Fremdsprachenunterricht. Tübingen: Francke, 299-302.

Spitzer, Manfred (2002): Lernen, Gehirnforschung und die Schule des Lebens. Heidelberg/Berlin: Spektrum.

Stier, Bernhard et al. (2005): Medienerziehung, Medienkompetenz, Medienpädagogik. Handreichungen und Informationen für Lehrer und Lehrerinnen sowie Hilfen und Unterstützung für den Unterricht. http://home.schule.at/teaching/reletik/modulmedienerziehung.pdf (letzter Zugriff: Zugriff: 23. 11. 2008)

Storch, Günther (1999): Deutsch als Fremdsprache. Eine Didaktik. Theoretische Grundlagen und praktische Unterrichtsgestaltung. München: Wilhelm Fink Verlag.

Thaler, Engelbert (2007a): Film-based Language Learning. In: Praxis Fremdsprachenunterricht, 1, 914.

Thaler, Engelbert (2007b): Schulung des Hör-Seh-Verstehens. In: Praxis Fremdsprachenunterricht, 4, 12-17.

Thaler, Engelbert (2010): Filmdidaktik. In: Hallet, Wolfgang/Königs, Frank (Hg): Handbuch Fremdsprachendidaktik. Seelze-Velber: Kallmeyer/Klett, 142-146.

Tschirner, Erwin (1997): Deutsch als Fremdsprache im Medienzeitalter. Fremdsprache Deutsch. In: Zeitschrift für die Praxis des Deutschunterrichts, Sondernummer II, 55-58.

Von Gottberg, Joachim (2007): Die Bedeutung des Fernsehens bei der Konstruktion des Weltbildes. In: Bildung und Erziehung, 4, 395-409.

Wandel, Reinhold (2002): Video, Marsmännchen und interkulturelles Lernen. In: Decke-Cornill, Helene/Reichart-Wallrabenstein, Maike (Hg.): Fremdsprachenunterricht in medialen Lernumgebungen. Frankfurt am Main: Peter Lang, 139-151.

Wegener, Claudia (2007): Stichwort: Medienforschung in der Erziehungswissenschaft. In: Zeitschrift für Erziehungswissenschaft, 4, 459-477. http://de.wikipedia.org/wiki/Orbis_sensualium_pictus (letzter Zugrift: Zugriff: 17.10.2009)

Wolff, Dieter (2004): Kognition und Emotion im Fremdsprachenerwerb. In: Börner/Vogel (Hg.): Emotion und Kognition im Fremdsprachenunterricht. Tübingen: Narr, 87-103.

Zeitlinger, Edith (2003): Unterhaltungsfilme im Unterricht? Versuch einer Begründung in Theorie und Praxis. In: Ide-Zeitschrift für den Deutschunterricht in Wissenschaft und Schule, 4, 78-85. 